\title{
VIVÊNCIAS COTIDIANAS DE ADOLESCENTES COM DIABETES MELLITUS TIPO 1
}

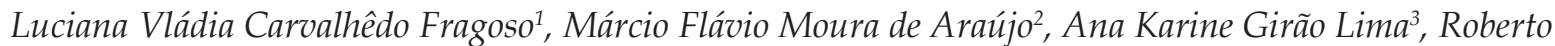 \\ Wagner Júnior Freire de Freitas ${ }^{4}$, Marta Maria Coelho Damasceno ${ }^{5}$
}

\footnotetext{
${ }^{1}$ Mestre em Enfermagem. Enfermeira do Hospital Universitário Walter Cantídio da Universidade Federal do Ceará. Ceará, Brasil. E-mail: luciana.vladia@hotmail.com

${ }^{2}$ Doutorando do Programa de Pós-Graduação em Enfermagem da Universidade Federal do Ceará (UFC). Professor Assistente do Curso de Enfermagem da Universidade Federal do Maranhão - Imperatriz. Maranhão, Brasil. E-mail: marciofma@yahoo.com.br

${ }^{3}$ Especialista em Unidade de Terapia Intensiva. Enfermeira do Hospital Geral de Fortaleza. Ceará, Brasil. E-mail: giraoenf@ gmail.com

${ }^{4}$ Mestre em Enfermagem. Professor Assistente do Curso de Enfermagem da Universidade Federal do Piauí - Floriano. Piauí, Brasil. E-mail: robertowjff@globo.com

${ }^{5}$ Doutora em Enfermagem. Professora do Programa de Pós-Graduação em Enfermagem da UFC. Pesquisadora do CNPq. Ceará, Brasil. E-mail: martadamasceno@terra.com.br
}

\begin{abstract}
RESUMO: Pesquisa qualitativa, cujo objetivo foi compreender as vivências cotidianas de adolescentes com diabetes do tipo 1. Os sujeitos foram constituídos por 14 adolescentes atendidos em um ambulatório de diabetes em Fortaleza, Ceará, Brasil, no período de junho a outubro de 2008. Os dados foram coletados através de entrevistas semi-estruturadas e organizados segundo a análise de conteúdo de Morse e Field. Observamos que a descoberta do diabetes foi um momento difícil para os adolescentes devido às mudanças que tiveram de adotar como controle terapêutico. Seguir a dieta adequada é algo bastante difícil em conseqüência dos estímulos internos e externos a que estão submetidos e as dificuldades financeiras. Os adolescentes têm como suporte terapêutico diário o apoio familiar e dos amigos. Além disso, eles gostam da assistência realizada pelos profissionais de saúde do ambulatório. Há muito que avançarmos enquanto enfermeiros na valorização das experiências de vida do adolescente com diabetes, identificando fatores que interferem no controle metabólico.
\end{abstract}

DESCRITORES: Acontecimentos que mudam a vida. Diabetes mellitus. Adolescente.

\section{DAILY EXPERIENCES OF ADOLESCENTS WITH TYPE 1 DIABETES MELLITUS}

\begin{abstract}
This descriptive, qualitative study aimed to better understand the type 1 diabetic adolescents' daily life experiences, per their speeches. The participants were 14 adolescents treated as diabetic outpatients in Fortaleza, Ceará, Brazil, from June to October of 2008. Data was collected through semi-structured interviews and organized according to Morse and Field content analysis. We observed that the discovery of diabetes was a difficult moment due to the changes they had to adopt as therapeutic control. Following the appropriate diet was seen to be a little bit difficult as a consequence of the internal and external motivation to which they are submitted and their financial difficulties. The adolescents have their families' and friends' support for their daily treatment. Furthermore, they like the service performed by ambulatory healthcare professionals. There is still a lot to improve as health professionals in valuing the adolescents' life experiences with diabetes, identifying factors that interfere with metabolic control.
\end{abstract}

DESCRIPTORS: Life change events. Diabetes mellitus. Adolescent.

\section{LAS EXPERIENCIAS COTIDIANAS DE LOS ADOLESCENTES CON DIABETES MELLITUS TIPO 1}

\begin{abstract}
RESUMEN: Investigación de carácter cualitativo, con el objetivo de comprender las experiencias cotidianas de los adolescentes con diabetes tipo 1. Los sujetos del estudio son 14 adolescentes atendidos en una clínica para la diabetes en Fortaleza, Ceará, Brasil, de junio a octubre de 2008. La recolección de los datos se hizo a través de entrevistas semi-estructuradas y se organizaron de acuerdo al análisis de contenido de Morse y Field. Se observó que el descubrimiento de la diabetes fue un momento difícil para los adolescentes debido a los cambios que tuvieron que hacer como el control terapéutico. Hacer una dieta adecuada es muy difícil como consecuencia de estímulos internos y externos a que están sometidos y a las dificultades financieras. Los adolescentes tienen el apoyo de la familia y amigos para su tratamiento diario. Además, les gusta el servicio realizado por los profesionales de salud de la clínica. Sin embargo, hay mucho que mejorar como enfermeras en la valorización de las experiencias de la vida de los adolescentes con diabetes, como la identificación de factores que interfieren en el control metabólico.
\end{abstract}

DESCRIPTORES: Acontecimientos que cambian la vida. Diabetes mellitus. Adolescente 


\section{INTRODUÇÃO}

A International Diabetes Federation (IDF) revela que a cada ano mais de 70 mil crianças e adolescentes desenvolvem Diabetes Mellitus do tipo 1 (DM 1). Diante deste quadro, a assistência ao adolescente com Diabetes Mellitus (DM) e suas famílias deve visar o viver mais saudável, indo além do conhecimento sobre as alterações físicas e psíquicas, mas é necessário também compreender as experiências construídas por essas pessoas no processo de viver com a doença. ${ }^{1}$

Dessa maneira, os profissionais encarregados de promover a saúde dos adolescentes devem fazer uso da visão positiva que os adolescentes possuem de si, tendo como meta subsidiá-los na aceitação de seus potenciais e limites e na capacidade de ousar a vida. ${ }^{2-3}$ Para tanto, é preciso ser conhecido suas histórias e experiências, o significado que atribui à saúde e à doença e sua correlação com os modos de vida. ${ }^{1}$

A descoberta do DM 1 em um adolescente requer além da incorporação de novos hábitos como o uso da insulina, realização de glicemia e a incorporação da atividade física diária, mas em especial perpassa pela aceitação da condição de portador de DM 1 que por vezes é percebida como uma doença que impõe limitações além das físicas, pois coloca a pessoa numa condição crônica pelo resto de sua vida. ${ }^{4}$

Então, estar aberto a compreender o viver do adolescente com diabetes é ponto fundamental para que se preste uma assistência eficaz e se propicie uma melhor qualidade de vida para o mesmo, minimizando os sentimentos negativos. Contudo, no âmbito hospitalar de muitas instituições públicas que atendem adolescentes diabéticos, percebemos que parte dos profissionais ainda permanecem voltados para o controle glicêmico, em detrimento dos aspectos existenciais, dicotomizando seu corpo entre ser diabético e ter diabetes, numa clara demonstração que o cuidado ainda é mais voltado para doença do que para o ser que a possue.

Todavia, muitos profissionais de saúde tentam compreender o que os adolescentes com DM 1 buscam para o seu tratamento e cuidado, através de ações mais humanizadas, centradas no respeito e na troca de saberes, numa relação mais horizontal. Tal aspecto ganha destaque inclusive em pesquisas. Dentre as encontradas três são destacáveis pelos seus achados.
A primeira teve como foco do estudo a percepção, compreensão e significados dos adolescentes sobre a experiência de ter DM. Os autores concluíram que compreender como o adolescente lida com o DM e saber identificar qual fase ele está vivenciando, é primordial para que a equipe de saúde possa propor intervenções que sejam realmente eficazes no cuidado desse adolescente. ${ }^{5}$

O segundo objetivou compreender o itinerário terapêutico de adolescentes com DM 1 e seus familiares. O problema de pesquisa emergiu a partir da seguinte constatação: apesar da prática a educação em saúde, relacionada essencialmente ao controle glicêmico, os jovens continuavam com suas glicemias alteradas. O que levou os profissionais de saúde a refletir que há um hiato entre o que se orienta e o que realmente é colocado em prática no cotidiano. Os resultados mostraram que o itinerário terapêutico se dá no subsistema familiar a partir do momento da aceitação e compreensão do diagnóstico e das recomendações terapêuticas, sendo a família nuclear a principal cuidadora desse adolescente. Os cuidados também são vinculados ao subsistema popular que incluem a busca pelo apoio espiritual e o uso de chás que são indicados pela comunidade na intenção de aliviar os sintomas da doença. ${ }^{1}$

No terceiro estudo os autores investigaram a dinâmica familiar no DM na infância e adolescência e perceberam que a existência de uma doença crônica desestrutura o contexto familiar, pois o cuidado prestado requer tempo, energia e pode provocar problemas sociais e emocionais entre os membros da família e no adolescente. Em contrapartida, se a família é bem orientada e recebe as informações necessárias para, junto com o jovem, exercer o cuidado eficaz, essa parceria entre pais e filhos fica reforçada e os resultados com a saúde do adolescente demonstram-se satisfatórios. Mas para isso necessitam do apoio nos serviços de saúde e dos profissionais através de uma assistência integral que abarquem suas carências, como também de uma rede social de apoio que os ofereça ajuda para enfrentar as dificuldades cotidianas. ${ }^{6}$

Outra constatação é que dentre a variedade de estudos que abordam o adolescente com DM 1 , poucos são aqueles que buscam uma apreensão da realidade de sua vida diária. Apesar da capacidade dos adolescentes de expor como lidam com a doença, suas dificuldades e mudanças cotidianas 
após o diagnóstico e como são cuidados no âmbito familiar, social e no serviço de saúde.

A literatura aponta ainda que o enfermeiro possui conhecimentos acerca das necessidades terapêuticas dos adolescentes com DM, mas em boa parte dos casos ele desconhece o significado das experiências vividas por eles e isso representa uma lacuna na assistência de enfermagem que passa a estabelecer um cuidado que não abarca o mundo do jovem diabético. Diante disso, a realização desse estudo tem como objetivo compreender as vivências cotidianas de adolescentes com DM 1.

\section{CAMINHO METODOLÓGICO}

Trata-se de um estudo descritivo de cunho qualitativo. A pesquisa foi desenvolvida no ambulatório de um hospital universitário de referência em atendimento aos portadores de diabetes, localizado na cidade de Fortaleza-CE. Na instituição, os adolescentes com DM recém-diagnosticados têm consultas mais freqüentes no serviço, com a melhora da saúde o acompanhamento normalmente acontece a cada três meses. A equipe de saúde era composta por endocrinologistas, um enfermeiro gerente e dois assistenciais, nutricionistas e técnicos de enfermagem.

Participaram do estudo 14 adolescentes com DM 1, com pelo menos um ano de tratamento, de ambos os sexos, procedentes do interior ou capital, na faixa etária de 12 a 18 anos. A opção por adolescente em tratamento para diabetes há pelo menos um ano ocorreu para auxiliar na obtenção de dados mais consistentes, pois haveria um tempo de experiência maior com a doença e um cenário com riqueza de informações, ambos requisitos importantes no delineamento qualitativo de pesquisa. ${ }^{7}$ A faixa etária foi delimitada com base no Estatuto da Criança e do Adolescente. ${ }^{8}$

O estudo foi realizado no período de junho à outubro de 2008, após autorização do Comitê de Ética em Pesquisa da instituição. Foram realizadas entrevistas semi-estruturadas com três perguntas norteadoras como forma de pré-teste com três jovens. Diante das limitações percebidas nessa fase novas questões foram acrescidas a fim de se obter mais dados acerca da experiência do adolescente em ter uma doença crônica como o DM 1.

Para coletar os dados se aguardava o comparecimento do adolescente na sala de procedimentos para coleta de exames laboratoriais, quando era explicado o objetivo da pesquisa ao mesmo e ao seu responsável e solicitado a assinatura do responsável ou do adolescente, caso tivesse idade $\geq 18$ anos, do Termo de Consentimento Livre e Esclarecido (TCLE). As entrevistas foram gravadas em aparelho MP 4, antes ou após o adolescente ter realizado sua consulta, salas privativas, para não prejudicar o fluxo do serviço e garantir o sigilo, a privacidade e a qualidade da gravação.

Os dados foram transcritos, logo após gravação, e submetidos a várias leituras flutuantes. Foi realizado um total de 14 entrevistas, pois, devido à saturação dos dados que não acrescentavam novas informações às já existentes nesse valor. Após a transcrição das 14 entrevistas, retornamos aos adolescentes entrevistados e as entrevistas foram validadas pelos mesmos sendo acrescidos ou esclarecidos alguns aspectos importantes.

Para a análise dos dados, foi considerado o referencial de Morse e Field que preconizam, na análise qualitativa de conteúdo, os seguintes passos: identificação, codificação e categorização dos dados. Isto implica que o pesquisador deve procurar o significado de passagens específicas e alocá-las em categorias apropriadas. ${ }^{9}$ Dessa maneira, foram elaboradas quatro unidades temáticas e cinco categorias, a saber:

A percepção do adolescente frente ao impacto de conviver com o diagnóstico de DM. Categoria - Ter que aprender a conviver com a doença.

Enfrentando uma dura realidade para seguir o controle do regime terapêutico. Categoria - Ter dificuldades para seguir a dieta.

O papel da família e dos amigos na cooperação do tratamento do adolescente com DM 1. Categorias - Ser cobrado e apoiado pela família para realizar o tratamento e Ter o apoio dos amigos como suporte para o tratamento.

O cuidado realizado pelos profissionais de saúde aos adolescentes com DM 1. Categoria Gostar da assistência prestada pelos profissionais de saúde no ambulatório de DM.

A pesquisa respeitou todos os preceitos éticos da pesquisa com seres humanos, conforme parecer $n^{\circ} .038 .0608$ do Comitê de Ética em Pesquisa com Seres Humanos (COMEPE) da instituição lócus da pesquisa. $\mathrm{O}$ anonimato dos sujeitos foi garantido através do estabelecimento de nomes próprios fictícios. 


\section{CONSTRUÇÃO DOS RESULTADOS}

A análise das falas permitiu a compreensão através de quatro temas e cinco categorias descritas a seguir, acerca da visão do adolescente sobre a sua experiência em conviver com o DM 1.

\section{A percepção do adolescente frente ao impac- to de conviver com o diagnóstico de DM 1}

\section{Ter que aprender a conviver com a doença}

Nesta categoria, ao falarem como se sentem convivendo com uma doença crônica como o diabetes, os entrevistados deram início aos seus discursos, relembrando como foi difícil o momento da descoberta do DM 1, devido às mudanças na rotina que tiveram que adotar, em virtude do controle terapêutico, porém, hoje vivem uma realidade melhor, apesar de algumas limitações. Por isso, se percebem como jovens normais. Vejamos as falas que nos comprovam essa nuance:

[...] no começo teve um pouco de dificuldade para me acostumar [...] porque tive que mudar bastante, hoje já sou mais acostumada [...]. Diabetes é uma coisa que você sabendo conviver, sabendo lidar você pode ter uma vida normal (Rebeca).

[...] pra mim é normal porque eu já tenho isso, eu encaro o meu dia-a-dia como algo assim normal que eu vou ter que passar por isso mesmo, é a minha rotina (Homero).

\section{Enfrentando uma dura realidade para seguir o controle do regime terapêutico}

\section{Ter dificuldades para seguir a dieta}

Esta categoria refere-se às dificuldades que os adolescentes enfrentam no seu cotidiano através dos seus desejos internos ou dos estímulos externos em torno da alimentação. Seguir a dieta adequada foi considerado pela maioria dos entrevistados como algo bastante difícil e que requer deles um controle muito grande. Por isso, às vezes, eles transgridem a dieta. Também emergiu nas falas a dificuldade financeira como dificultador da dieta correta, ou seja, há vontade de se alimentar melhor, mas a renda familiar não é suficiente para suprir suas necessidades nutricionais. As falas seguintes comprovam isso:

[...] às vezes dá vontade da gente comer eu num vou dizer que eu não como porque se eu disser que eu não como eu estou mentindo, às vezes eu como assim escondido, mas não é todo dia é de vez em quando, quando bate aquela vontade que você não pode segurar, a gente come (Gabriela).

[...] tenho bastante dificuldade com relação à alimentação porque por eu ser adolescente e ser do meio onde tem muito doce, muito refrigerante, muita coisa que eu não poso comer, então eu tenho dificuldade nisso, o meu maior problema é em relação à alimentação, mas no resto eu convivo bem com o diabetes (Rebeca).

[...] eu gosto muito de salgado, eu fujo da dieta eu como bastante o grosso arroz, feijão, macarrão para não ficar com fome. Eu não tenho condições de me alimentar como um diabético porque o dinheiro não dá (Lídia).

\section{O papel da família e dos amigos na coo- peração do tratamento do adolescente com DM 1}

\section{Ser cobrado e apoiado pela família para rea- lizar o tratamento}

Nesta categoria os jovens destacam que a família tem um papel fundamental no tratamento do DM 1. Os adolescentes percebem isto através das cobranças familiares no dia-a-dia para que as ações de autocuidado sejam realizadas. Apesar dos jovens ficarem, por vezes, chateados com essas cobranças, eles ainda assim percebem que essa é uma atitude de zelo e para o bem deles, como mostram os depoimentos.

É um pouco difícil, a pessoa é muito cobrada pelos familiares, e eles, às vezes [...] não entendem [...] o que realmente a gente sente porque é uma coisa muito difícil, é uma transformação muito difícil na nossa vida $e$ às vezes as pessoas tem muitas barreiras, mas eles, às vezes, não entendem. Mas nós sabemos que essa preocupação deles é pro nosso bem eles estão sempre querendo melhorar, sempre nos ajudando e as vezes nós é que não seguimos o que deve ser feito, nós não cumprimos a nossa tarefa e eles tem que cobrar, é obrigação deles, mas às vezes a gente leva como se fosse uma forma de implicar com a gente, mas com certeza eles fazem isso para o nosso bem (Rute).

É assim eu tenho o apoio da minha família sempre que eu vou fazer alguma coisa com meus irmãos, minhas tias sempre eles cuidam muito de mim sempre tenho o apoio da minha mãe, da minha família toda aí assim o apoio é sempre que eu tenho (Eunice).

\section{Ter o apoio dos amigos como suporte para o tratamento}

Essa categoria nos revela que além do apoio da família, os adolescentes com DM 1 contam tam- 
bém com o suporte dos amigos para enfrentarem o tratamento. Vejamos as falas dos entrevistados para corroborar com isso:

[...] todos os meus amigos e as pessoas que eu me relaciono sabem que eu sou diabética e eles também me ajudam bastante evitando que eu coma doce, evitando comer doce na minha frente, também chamam a minha atenção quando me vêem querendo comer alguma coisa doce, algo que eu não posso comer, eles chamam a minha atenção e me ajudam bastante (Rebeca).

[...] eles me tratam normal, como qualquer outra pessoa que não tenha essa doença, saio com eles, mas eles também se preocupam comigo, pensam na hora que vão voltar pra não sair do horário de aplicar a insulina, então eles me ajudam muito em relação a isso (Homero).

\section{O cuidado realizado pelos profissionais de saúde aos adolescentes com DM 1}

\section{Gostar da assistência prestada pelo médico e enfermeiro do ambulatório de diabetes}

Essa categoria mostra a satisfação dos adolescentes com o atendimento que eles recebem no serviço de saúde. Os discursos revelaram satisfação com a assistência prestada pelos médicos do setor de endocrinologia, porém, notamos que o cuidado ainda é bastante voltado para o suprimento das necessidades biológicas do diabetes. Emergiu-se das falas que o cuidado de enfermagem atendia às necessidades emocionais e de adesão ao tratamento.

Vejamos os discursos:

[...] eu gosto do atendimento aqui no hospital dão o que eu preciso o remédio, a insulina, dão as seringas, sou bem atendida sou monitorada todo mês, os médicos perguntam tentam me ajudar também fazendo os exames, tudo eu faço aqui e gosto do atendimento [...] seria bom também não só o endocrinologista, mas também ter vários outros médicos porque a diabetes com o tempo pode levar a outras doenças (Rebeca).

[...] quando eu me internei aqui ai eu tive o atendimento de uma enfermeira, ela me ensinou como aplicar a insulina como preparar a insulina como administrar se ela tiver baixa alta ela sempre me ensinou. Ela me ensinou também a encarar a vida, [...] me ajudou me deu muito conselho [...] (Eunice).

[...] é bom o atendimento dos médicos, dão sempre conselhos como evitar comer muitas coisas gordurosas, evitar comer açúcar [...] porque como eu tenho quatorze anos é muito difícil [...] ficar sem açúcar. Então eles sempre me dão conselhos para fazer uma dieta saudável então é bom o atendimento aqui (Homero).

\section{SÍNTESE COMPREENSIVA}

Identificamos nas falas da primeira categoria que os adolescentes sentiram-se impactados diante do diagnóstico devido às mudanças que tiveram que inserir no seu cotidiano através do aprendizado de novas condutas com a sua saúde, porém o tempo fez com eles aprendessem a conviver e a se acostumarem com a nova vida adquirindo habilidades para lidar com DM 1, passando então a se sentirem como uma pessoa normal que realiza atividades comuns a outros adolescentes.

Outros autores, ao pesquisar sobre o cotidiano e o enfrentamento da doença por adolescentes diabéticos, encontraram modo de pensar semelhante ao expressado no presente estudo: Apesar do início da doença ser avaliado como de considerável dificuldade, o tempo de convivência fez com que as dificuldades diminuíssem, não trazendo mais tantos transtornos, e possibilitando uma diminuição no nível de estresse experimentado pelo paciente crônico. ${ }^{10}$

Os adolescentes gostam de sentirem-se iguais aos seus pares; eles buscam constantemente viver dentro da normalidade apregoada pela sociedade por isso apesar de conviver com limitações no seu cotidiano advindas do controle metabólico do DM 1, demonstraram com suas falas que estão buscando enfrentar e seguir o seu tratamento e continuar vivendo como pessoas normais. Portanto, as estratégias de enfrentamento utilizadas por eles são encarar a sua rotina, apreender a lidar com as contingências do diabetes e seguir o tratamento corretamente.

Pesquisadores, ao estudarem adolescentes diabéticos, observaram, nos seus discursos que os mesmos são capazes de analisar suas experiências iniciais com o diabetes como difícil, mas ao relacionar como lidam no presente com seu tratamento, concluem que hoje estão mais instrumentalizados, sendo por isso mais fácil conviver no cotidiano com todas as exigências que o diabetes requer. ${ }^{5}$ Por sua vez, outros estudiosos encontraram depoimentos que relataram que a aceitação da doença foi difícil devido aos obstáculos no cotidiano, como a dificuldade para preparar alimentos apropriados para o diabético e, por fim, alguns expressaram que é preciso possuir esperança e espírito de coragem para conviver melhor com as dificuldades que DM acarreta. ${ }^{11}$ 
Nos discursos dos adolescentes desse estudo observamos significados semelhantes ao estudo supracitado, quando eles verbalizam que tiveram que se acostumar com as mudanças, que se sentem como pessoas normais buscando uma harmonia com a doença crônica e que ser diabético é enfrentar e encarar o tratamento, ou seja, é possuir espírito de coragem.

Os discursos da segunda categoria também demonstram que seguir a dieta é difícil por diversos motivos como viverem num meio onde existe muito doce, refrigerante, por gostarem de alimentos salgados e carboidratos, de terem que se alimentar em horários determinados e também conviverem no seu lar com guloseimas como bolo, chocolate, etc. Parece que aquilo que é proibido realmente é o que chama mais atenção do adolescente. Até porque, nessa fase, viver sob risco e quebrar regras é uma forma de amadurecimento para eles; então, manter-se numa dieta saudável e equilibrada é um problema dentro do mundo do adolescente diabético.

Acerca disso, uma pesquisa estudou a vivência de tornar-se portador de DM 1 e identificou que a restrição alimentar, devido às exigências terapêuticas, e as dificuldades de não alimentarse como os amigos não diabéticos, são fatores que impactam no cotidiano do adolescente com DM 1. Os principais fatores mencionados foram às limitações na ingestão de doces quando na presença dos amigos na hora do lazer e não poder mais comer alimentos doces na escola. ${ }^{12}$ Outros autores reforçam este discurso e argumentam que a dieta alimentar representa um dos principais aliados do tratamento, entretanto, mas também o maior vilã, devido à dificuldade de aceitação da restrição alimentar frente aos apelos sociais de fast foods, bebidas e doces, tornando-se, assim, um grande desafio. ${ }^{13}$

Os adolescentes com diabetes vivem em conflito, pois sabem da importância de não quebrar as regras do seu tratamento, mas, em contrapartida, o desejo de comer outros alimentos, às vezes, se torna incontrolável e, como eles próprios verbalizaram, dão uma "escapadinha". Por isso é preciso dar oportunidade para que os adolescentes demonstrem realmente o que sentem e passam em suas vidas em decorrências de ter que reprimir suas vontades em diversas situações do convívio social.

Foi observado também, nas falas dos adolescentes entrevistados na terceira categoria, que o suporte dos familiares é importante, pois os ajudam a realizar o tratamento diário, mesmo que para isso tenham que ser lembrados constantemente.

Ao estudar as estratégias de enfrentamento dos adolescentes com DM 1, pesquisadores encontraram que o suporte e apoio social são estratégias usadas pelos jovens como um fator positivo para enfrentarem o efeito indesejado do estresse a doença. Os mesmos buscam conversar com os familiares na tentativa de aceitar a realização de um tratamento diário e repetitivo, pois, muitas vezes, sentem-se esgotados por terem que fazer sempre as mesmas coisas todos os dias, mas ao mesmo tempo lembram-se do zelo e do cuidado da família que os impulsionam a avançar e enfrentar as dificuldades. ${ }^{14}$

Pesquisa bibliográfica sobre os aspectos emocionais vivenciados pelos diabéticos identificou que a família influencia no seguimento do tratamento. A partir da análise dos estudos ficou claro pacientes com DM que possuem apoio familiar adequado, aderem melhor às orientações de autocuidado, demonstrando a importância da aceitação da doença pelo portador de DM mediante o apoio familiar.${ }^{15} \mathrm{Em}$ Portugal, um estud o com adolescentes insulino-dependentes investigou variáveis psicossociais na predição de melhores resultados terapêuticos. Ficou evidenciado que para se ter um melhor controle metabólico é necessário receber apoio familiar e dos profissionais de saúde relativo à doença, através de comportamentos de apoio instrumental e emocional por meio de palavras de ânimo e encorajamento. Dessa forma, os profissionais de saúde e a família são determinantes num melhor controle do DM. ${ }^{16}$

Dados semelhantes aos estudos supracitados são encontrados entre os entrevistados deste estudo. Aqui, os jovens percebem isto como um fator relevante, pois através do apoio instrumental e emocional é que eles vão se adaptando às rotinas e transpondo os obstáculos que surgem no seu cotidiano. Ainda na terceira categoria percebemos que como a família os amigos também são elos fortes e importantes no cotidiano do adolescente diabético, pois eles exercem papel semelhante ao da família, encorajando e dando apoio na realização do tratamento.

Tanto a família como os amigos são destacados também em outros estudos com adolescentes portadores de DM 1, como suporte de apoio e incentivo frente às limitações e terapêutica desta endocrinopatia. ${ }^{10,12}$ Especificamente acerca das amizades de adolescentes com diabetes, a literatura mostra que esse apoio envolve o fator 
emocional que está relacionado ao fato dos amigos não tratarem com diferença os colegas diabéticos e ainda tentarem tranqüilizá-los acerca da doença. Os amigos se envolvem ainda na adequação dos comportamentos dos amigos à condição dos mesmos e buscando informações para lidar com o DM 1 e para atualizar o amigo. ${ }^{17}$

Outro ponto destacável é o fato de o adolescente ter DM não interferir em seus relacionamentos de amizade. ${ }^{5}$ Nesta investigação todos os adolescentes disseram que os seus amigos mais próximos são os que com eles conversam sobre o DM 1. Na literatura há relatos que atribuem ser importante o suporte dos amigos na tentativa de ajustar o adolescente à sua doença e a saber conviver com ela e, ainda, afirma que a presença dos amigos proporciona ao adolescente o elo que lhe assegura os vínculos com o "seu mundo", dando-lhe uma sensação de ainda pertencer a esse mundo. ${ }^{18}$

Nas falas dos adolescentes da quarta categoria identificamos que a satisfação com atendimento no serviço de saúde está relacionada ao bom tratamento que eles recebem dos médicos e enfermeiros, pela educação em saúde voltada para o controle do diabetes, através do ensino para o autocuidado e pela atitude de paciência dos profissionais no repasse de informações importantes para o manejo do DM 1.

Em concordância com esse achado, há publicações que, ao estudarem como o ser humano diabético percebe o cuidado de enfermagem, encontraram que "cuidar" é demonstrar carinho, paciência, tratar bem e atender às necessidades, quer físicas ou biológicas. ${ }^{19}$ Nesse sentido, a prática educativa da enfermeira é representada também pelos adolescentes como mãos que apóiam nos momentos difíceis com carinho e conforto. E, ainda, foi relatado pelos mesmos que o enfermeiro é como uma extensão da família ${ }^{13}$. Por sua vez, o contentamento relacionado ao tratamento médico é de $92,85 \%$ numa investigação brasileira. ${ }^{20}$

Os adolescentes entrevistados também declararam gostar do atendimento que receberam devido às orientações concernentes ao automanejo do DM 1, envolvendo cuidados com a alimentação e insulinoterapia. A literatura, acerca deste ponto, revela que a satisfação do paciente diabético nas consultas está relacionada com as informações recebidas em temas como dieta $(59,3 \%)$, medicamentos $(33,3 \%)$ e controle glicêmico $(31,5 \%)$. Outro destaque também é a excelência do atendimento. ${ }^{21}$
A ausência de uma equipe multiprofissional leva à vulnerabilidade dos serviços de saúde para diabéticos, no que diz respeito à adoção de um estilo de vida saudável. Assim, a atuação da equipe é fundamental para uma melhor qualidade na assistência ao paciente com DM..$^{22,23}$

Infelizmente, poucos municípios brasileiros possuem centros especializados com uma equipe multiprofissional para o atendimento do diabetes mellitus e tão pouco esses sujeitos ficam resignados aos ambulatórios. Eles também estão presentes em unidades básicas, hospitais, asilos e centros de reabilitação, portanto, é importante que todo contato entre profissionais de saúde e os diabéticos nestes ambientes seja utilizado para a prática da educação em saúde na perspectiva do controle metabólico da doença.

Nas falas dos adolescentes deste estudo, por exemplo, pode se observar que médicos e enfermeiros procuram ter atitudes como dar conselhos, tirar as dúvidas, ter paciência no repasse das informações, fornecer livros informativos e dar suporte emocional. Essas atitudes positivas utilizadas pelos profissionais durante o atendimento dos adolescentes apresentam-se como pontos importantes e que propiciaram um contentamento com o serviço de saúde.

\section{CONSIDERAÇÕES FINAIS}

Será que conhecer a realidade cotidiana do adolescente com DM 1 contribui com o melhor manejo do seu tratamento? Sim, conhecer a realidade vivida pelos adolescentes com DM 1 e compreender suas experiências cotidianas nos permitiu identificar alguns aspectos que contribuirão de maneira positiva ou negativa para o manejo do tratamento do DM. Através dos discursos dos adolescentes percebemos suas dificuldades ou facilidades para lidar com uma doença crônica diariamente.

Os discursos revelaram que para nos apropriarmos de um cuidado com qualidade é preciso ouvir os adolescentes, para assim podermos construir uma assistência que valorize seus sentimentos, atitudes, relações sociais, culminando assim em adolescentes competentes para o autocuidado, e que por isso terão poucas complicações em decorrência do DM, por receberem assistência profissional e familiar integral com vistas à promoção de sua saúde. Mas, para isso, é necessária uma remodelagem na forma de assistir ao adolescente com DM 1, priorizando suas necessidades e planejando juntamente com eles e seus familiares ações a 
curto, médio e longo prazo que contribuam para a sua qualidade de vida e bom controle glicêmico.

Essa pesquisa não representa a totalidade do universo do ser diabético, entretanto, nos mostrou aspectos importantes, dentre os quais: o suporte familiar, dos amigos e dos profissionais de saúde na cooperação com o tratamento do adolescente com DM 1 no cotidiano.

Faz-se relevante que as pesquisas realizadas sobre as experiências vividas pelos adolescentes com diabetes, assim como essa, sejam colocadas em prática pelos profissionais de saúde que atendem a essa clientela nos ambulatórios de endocrinologia, com o intuito de prestarem uma assistência que supram as necessidades que os mesmos têm diante das vivências no cotidiano social, familiar e nos serviços de saúde, já que alguns fatos evidenciados nesse estudo são semelhantes aos achados de estudos realizados anos anteriores com esses sujeitos, demonstrando que a práxis profissional tem se modificado lentamente, precisando verdadeiramente de um aprimoramento na qualidade do atendimento aos adolescentes com DM 1.

\section{REFERÊNCIAS}

1. Mattosinho MMS, Silva DMGV. Itinerário terapêutico do adolescente com diabetes mellitus tipo 1 e seus familiares. Rev Latino-am Enfermagem. 2007 NovDez; 15(6):1113-9.

2. Assis SG. A representação social do ser adolescente: um passo decisivo na promoção da saúde. Ciênc Saúde Coletiva. 2003 Jul-Set; 8(3):669-79.

3. Cardoso MVLML, França DF, Sousa WR, Lúcio IML. Analysis of the publications about child health in journals of nursing from 2000 to 2005 . Online Braz J Nurs [online]. 2008 [cited 2008 Fev 22]; 7(1). Available from: http://www.uff.br/objnursing/index.php/ nursing/article/view/j.1676-285.2008.1168/304

4. Mello MB. Déficits de autocuidado vivenciados por famílias de crianças e adolescentes com diabetes mellitus tipo 1 [dissertação]. Florianópolis (SC): Universidade Federal de Santa Catarina, Programa de Pós-graduação em Enfermagem; 2006.

5. Damião EBC, Pinto CMM. Sendo transformado pela doença: a vivência do adolescente com diabetes. Rev Latino-am Enfermagem. 2007 Jul-Ago; 15(4):52-7.

6. Nunes MDR, Dupas G, Ferreira NMLA. Diabetes na infância/adolescência: conhecendo a dinâmica familiar. Rev Eletr Enferm [online]. 2007 [acesso 2008 Fev 22]; 9(1):119-30. Disponível em: www.fen. ufg.br/revista/v9/n1/pdf/v9n1a09.pdf

7. Polit DF, Beck KCT, Hungler BP. Fundamentos de Pesquisa em Enfermagem - métodos, avaliação e utilização. 5a ed. Porto Alegre (RS): Artmed, 2004.
8. Ministério da Saúde (BR). Estatuto da criança e do adolescente. $3^{\mathrm{a}}$ ed. Brasília (DF): Editora do Ministério da Saúde; 2008.

9. Manning PK, Cullum S. Betsy narrative, content and semiotic analysis. In: Denzin N, Lincoln YS. Handbook of qualitative research. New Delhi (IN): Sage Publications; 1994.

10. Santos JR, Enumo SRF. Adolescentes com diabetes mellitus tipo 1: seu cotidiano e enfrentamento da doença. Psicol Reflex Crit. 2003 Mai-Ago; 16(2):411-25.

11. Delmar C, Boje T, Dylmer D, Forup L, Jakobsen C, Moller M, Sonder H, Pedersen B. Achieving harmony with oneself: life with a chronic illness. Scand J Caring Sci. 2005 Sep; 19(3):204-12.

12. Imoniana BBS. Crise de identidade em adolescentes portadores de diabetes mellitus tipo 1. Psicol Am Lat [online]. 2006 Ago [cited 2010 Ago 8]; 7.Avaliable from: http://pepsic.bvsalud.org/ scielo.php?script=sci_arttext\&pid= S1870350X2006000300004\&lng=pt\&nrm=iso

13. Freitas FV, Sabóia VM. Vivências de adolescentes diabéticos e contribuições da prática educativa da enfermeira. Rev Enferm UERJ 2007 Out-Dez; 15(4):569-73.

14. Damião EBC, Dias VC, Fabri LRO. O adolescente e o diabetes: uma experiência de vida. Acta Paul Enferm 2010 Jan-Fev; 23(1):41-7

15. Marcelo DB, Carvalho MDD. Reflexões sobre o diabetes tipo1 e sua relação com o emocional. Psicol Reflex Crit. 2005 Jan-Abr; 18(1):72-7.

16. Almeida V, Matos AP. A diabetes na adolescência: um estudo biopsicossocial. Rev Int Psicol Clín Salud. 2003 Jan-Abr; 3(1):61-76.

17. Ferreira BES, Garcia A. Aspectos da amizade de adolescentes portadores de diabetes e câncer. Estud Psicol. 2008 Abr-Jun; 25(2):293-301.

18. Brito TB, Sadala MLA. Diabetes mellitus juvenil: a experiência de familiares de adolescentes e préadolescentes. Ciênc Saúde Coletiva. 2009 Mai-Jun; 14(3):947-60.

19. Dias DG, Santana MG, Santos E. O ser humano diabético frente ao cuidado humanizado. Rev Bras Enferm. 2006 Mar-Abr; 59(2):168-71.

20. Barros ACM, Rocha MB, Helena ETS. Adesão ao tratamento e satisfação com o serviço entre pessoas com diabetes mellitus atendidas no PSF em Blumenau, Santa Catarina. Arq Catarin Med. 2008 Jan-Mar; 37(1):54-62.

21. Zanetti ML, Otero LM, Peres DS, Santos MA, Guimarães FPM, Freitas MCF. Evolução do tratamento da pacientes diabéticos utilizando o protocolo staged diabetes management. Acta Paul Enferm. 2007 Jul-Set; 20(3):338-44.

22. Araújo MFM. Cumprimento da terapia com antidiabéticos orais entre usuários da rede básica 
de saúde de Fortaleza-CE [dissertação]. Fortaleza (CE): Universidade Federal do Ceará, Programa de Pós-graduação em Enfermagem; 2009.
23. Francioni FF, Silva DGV. O processo de viver saudável de pessoas com Diabetes Mellitus através de um grupo de convivência. Texto Contexto Enferm. 2007 Jan-Mar; 16(1):105-11. 